\section{Sintomas psicológicos concomitantes à queixa de vertigem em 846 prontuários de pacientes otoneurológicos do Ambulatório de Otoneurologia da Universidade Federal de São Paulo - Escola Paulista de Medicina}

\author{
Angela Daou Paiva', Ana Maria Baccari Kuhn'
}

Resumo

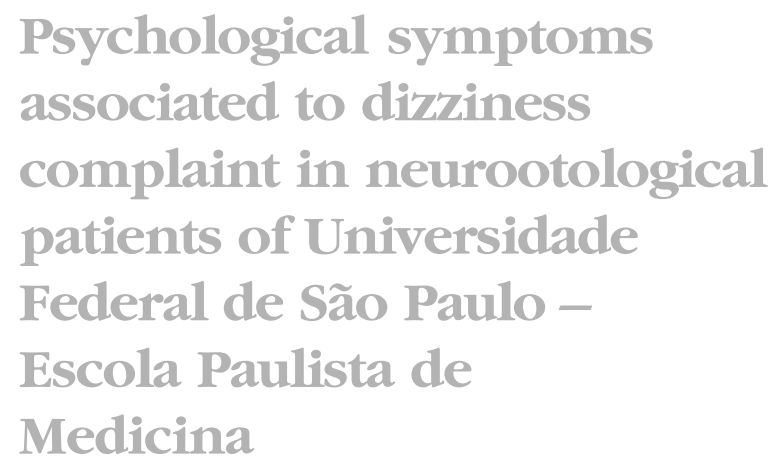

Palavras-chave: vertigem, sintomas psicológicos, angústia. Key words: dizziness, psychological symptoms, anguish.

Summary

$\mathbf{O}$ bjetivo: Verificar os sintomas psicológicos mais freqüentemente associados à queixa de vertigem de acordo com os registros de prontuários de pacientes otoneurológicos. Forma de estudo: Clínico retrospectivo. Método: Foram quantificados os dados relativos a sexo, idade e sintomas psicológicos, de acordo com os registros de 846 prontuários de triagem de pacientes otoneurológicos com queixa de vertigem, atendidos pelo Setor de Triagem do Ambulatório de Otoneurologia e Otoneuropsicologia da Universidade Federal de São Paulo - Escola Paulista de Medicina, nos períodos de 1999 a 2001. Resultados: A concomitância de sintomas psicológicos à queixa de vertigem foi verificada em 477 (56,38\%), de um total de 846 prontuários. Os sintomas psicológicos associados à queixa de vertigem foram a angústia (47,38\%), a ansiedade (19,71\%), o medo (13,42\%) a depressão (12,58\%) e os distúrbios de memória (6,92\%). Conclusão: A angústia foi o sintoma psicológico concomitante à queixa de vertigem de maior prevalência $(47,38 \%)$. im: To report the psychological symptoms associated to dizziness complaint in neurootological patients. Study design: Chart review. Method: A total of 846 medical reports of neurootological patients with dizziness complaint were quantified, concerning to gender, age and psychological symptoms associated to dizziness complaint. Results: The psychological symptoms associated to dizziness complaint were anguish (47,38\%), anxiety $(19,71 \%)$, fear $(13,42 \%)$, depression $(12,58 \%)$, and memory disturbances (6,92\%). Conclusion: Anguish $(47,38 \%)$ was the most prevalent psychological symptom associated to dizziness complaints in neurootological patients.

\footnotetext{
${ }^{1}$ Psicóloga, Psicoterapeuta, Mestranda em Distúrbios do Desenvolvimento pela Universidade Presbiteriana Mackenzie, Especialista em Otoneuropsicologia pela Universidade Federal de São Paulo. ${ }^{2}$ Psicóloga, Psicanalista, Professor Associado Livre-Docente, Chefe do Setor de Otoneuropsicologia Departamento de Otorrinolaringologia e Cirurgia de Cabeça e Pescoço Universidade Federal de São Paulo - Escola Paulista de Medicina. Endereço para correspondência: Ângela Daou Paiva - Rua Alberto Faria, 1349 Alto de Pinheiros 05459-001 São Paulo SP. 


\section{INTRODUÇÃO}

Muitos são os sintomas que podem estar associados às queixas de vertigem e a outros tipos de tontura. Dentre eles, os sintomas otoneurológicos (zumbido, hipoacusia, otalgia, cefaléia e náusea, por exemplo) e psicológicos (ansiedade, depressão e medo). Quaisquer alterações clínicas concomitantes devem ser consideradas como possíveis causas da vestibulopatia e, havendo diversas afecções simultâneas, todas devem estar implicadas como possíveis fatores etiológicos ${ }^{1,2,3}$. Alguns sintomas psicológicos podem ser causa, conseqüência ou coexistirem com as crises de vertigem, sob quatro aspectos: a) um transtorno psíquico (ansiedade, por exemplo) pode provocar uma crise; b) um transtorno psíquico, usualmente a depressão ou o transtorno do pânico, pode ser provocado ou causado por uma crise; c) um transtorno psíquico pode agravar uma crise e d) um transtorno psíquico pode estar representado por uma crise ${ }^{4}$.

Dentre os fatores psicológicos, a depressão, o medo, o pânico e a ansiedade são apontados pela literatura como os principais sintomas concomitantes à vertigem. Em estudo realizado com 75 pacientes vertiginosos, a depressão e o pânico foram descritos como parte do diagnóstico diferencial, salientando que esses apresentaram também índices mais elevados para transtornos psicológicos, bem como episódios depressivos recorrentes 5 .

A influência de fatores psicológicos nas disfunções vestibulares tem sido considerada no diagnóstico de pacientes vertiginosos. De 206 pacientes com queixa de vertigem, o grau de ansiedade, depressão e medo, especialmente para o sexo feminino, foi significativamente mais elevado ${ }^{6}$. A ansiedade foi descrita como sintoma comórbido da vertigem, cuja influência pode tornar o prognóstico menos positivo, com declínio na qualidade de vida de pacientes otoneurológicos ${ }^{7}$. A associação da ansiedade e da depressão aos distúrbios otoneurológicos foi apontada em um grupo de 91 pacientes com disfunções labirínticas e vestibulares, salientando a importância em detectar quais as implicações psicológicas concomitantes ${ }^{8}$. A ansiedade e o medo, bem como os distúrbios de memória, são descritos como fatores que podem tanto agravar as crises, quanto retardar a recuperação em pacientes otoneurológicos com queixa de vertigem? .

As crenças subjetivas (psíquicas) aos sintomas otoneurológicos podem contribuir negativamente para a recuperação de pacientes vertiginosos, como salienta o estudo realizado em 101 pacientes com disfunções vestibulares, em que foram identificados três grupos de crença: preocupação com a perda de controle (fator desencadeante de ansiedade e depressão), medo e antecipação de uma cri$\mathrm{se}^{10}$. A relação entre estabilidade emocional (ansiedade e medo) e manutenção do equilíbrio corporal foi demonstrada em estudo comparativo entre três grupos: a) com queixa de vertigem/desequilibrio; b) com relato de episódios verti- ginosos, mas sem queixa; c) sem queixas e/ou episódios vertiginosos ${ }^{11}$. A influência da agorafobia na eficácia da reabilitação vestibular em pacientes vertiginosos apontou para a concomitância e comorbidade de sintomas ${ }^{12}$.

A otoneuropsicologia considera a vertigem como uma das principais manifestações da angústia, como seu equivalente somático. As manifestações de angústia, bem como de ansiedade, fobia, depressão e medo merecem especial atenção no diagnóstico, terapêutica e prognóstico otoneurológico $^{13}$. As crises de vertigem são acompanhadas de angústia e as manifestações vertiginosas têm estreita ligação com as neuroses e sua natureza, apontando íntima correlação entre angústia e suas implicações no processo somático $^{14}$. O limite entre a angústia e a ansiedade é tênue. Enquanto a ansiedade refere-se à vivência de sofrimento psiquico, a angústia é entendida como um estado afetivo abrangente que engloba a ansiedade e as manifestações somáticas (alterações neurovegetativas) decorrentes desse sofrimento. As alterações neurovegetativas decorrentes do desequilíbrio corporal são nitidamente semelhantes às das crises de angústia e de pânico ${ }^{13,15}$.

Os sintomas psicológicos - depressão, ansiedade e medo - são apontados pela literatura como sintomas comórbidos da vertigem, cuja influência pode tornar o prognóstico menos positivo, com declínio na qualidade de vida de pacientes. Esta comorbidade sugere que estudos sejam feitos no sentido de determinar quais os principais quadros psicológicos concomitantes à queixa de vertigem em pacientes oteneurológicos. A angústia, como o equivalente psíquico da vertigem, merece ser, bem como a ansiedade, a depressão e o medo, investigada como possível sintoma psicológico concomitante à vertigem e considerada no diagnóstico, prognóstico e terapêutica de pacientes otoneurológicos.

\section{OBJETIVO}

O objetivo deste estudo foi o de verificar os sintomas psicológicos mais freqüentemente associados à queixa de vertigem em 846 prontuários de pacientes otoneurológicos, atendidos pelo Setor de Triagem do Ambulatório da Disciplina de Otoneurologia e do Setor de Otoneuropsicologia da Universidade Federal de São Paulo - Escola Paulista de Medicina, nos períodos de 1999 a 2001.

\section{MÉTODO}

Foram utilizados 846 prontuários - 322 de janeiro a dezembro de 1999, 238 de janeiro a dezembro de 2000 e 286 de janeiro a outubro de 2001 - de pacientes com queixa de vertigem, do Setor de Triagem do Ambulatório da Disciplina de Otoneurologia e do Setor de Otoneuropsicologia do Departamento de Otorrinolaringologia e Cirurgia de Cabeça e Pescoço da Universidade Federal de São Paulo 
- Escola Paulista de Medicina. As queixas foram registradas de acordo com o relato dos pacientes. Os prontuários foram preenchidos por equipe multidisciplinar, composta por profissionais da Otorrinolaringologia, Otoneurologia, Fonoaudiologia e Psicologia, em conformidade com o regulamento de conduta ética do Comité de Ética da Universidade Federal de São Paulo - Escola Paulista de Medicina.

A seleção, os critérios de inclusão, exclusão e a quantificação dos dados registrados nos prontuários foram efetuadas em quatro etapas: triagem, agrupamento, inclusão/exclusão e quantificação. Na primeira etapa, foram qualificadas todas as informações registradas nos prontuários. $\mathrm{Na}$ segunda etapa, essas informações foram agrupadas em nove categorias: 1) sexo, 2) idade, 3) queixas otoneurológicas (associadas e/ou não à queixa de vertigem), 4) doenças associadas, concomitantes e/ou anteriores, 5) medicação em uso, 6) histórico de cirurgias anteriores, 7) hábitos alimentares, 8) prática de tabagismo e 9) queixas associadas (psicológicas, cardiovasculares, metabólicas e hormonais). Na terceira etapa, foram excluídos os prontuários de pacientes otoneurológicos sem queixa de vertigem. Do total de 1041 prontuários, foram selecionados e incluídos $846 \mathrm{em}$ que a vertigem foi registrada como queixa principal (associada e/ ou não a outras sintomas otoneurológicos). Na etapa final, foram quantificados, em valores absolutos e relativos, somente os dados obtidos das categorias 1, 2, 3 e 9. Da categoria 9, foram incluídos somente os sintomas psicológicos associados, excluindo-se quaisquer outros (cardiovasculares, metabólicos e hormonais). Foram incluídos na categoria 9 somente os prontuários em que foram registradas queixas psicológicas e excluídos aqueles em que não houve o registro, o que não significa que esses pacientes não apresentaram queixas psicológicas, mas somente que essas queixas não foram registradas nos respectivos prontuários.

Do total de 846 prontuários selecionados, em 8 não foram encontrados registros de idade. Os dados relativos à idade foram agrupados por faixas etárias, em função de sua amplitude, considerando-se como categoria "não consta", os registros não encontrados. Na categoria de "sintomas psicológicos" foram considerados: a) angústia, b) ansiedade, c) depressão, d) medo e e) distúrbios de memória. Os critérios de inclusão para "angústia" e "ansiedade" foram definidos de acordo com a teoria psicanalítica.

\section{RESULTADOS}

Do total de 846 prontuários com queixa de vertigem, $612(72,34 \%)$ foram do sexo feminino e $234(27,66 \%)$ do masculino, com idades entre 20 e 80 anos. A faixa etária de menor prevalência foi a de 20 a 30 anos (11\%), sendo $69(74,2 \%)$ prontuários do sexo feminino e $24(25,8 \%)$ do masculino. De 31 a 40 anos, foram registrados 120 (14,2\%) prontuários, sendo $85(70,9 \%)$ do sexo feminino e $35(29,1 \%)$ do masculino. O total de prontuários na faixa etária de 41 a
50 anos foi de 207 (24,47\%), sendo 145 (70,1\%) do sexo feminino e $62(29,9 \%)$ do masculino. A faixa etária de maior prevalência foi de 51 a 60 anos (25,3\%), com $150(70 \%)$ prontuários do sexo feminino e 64 (30\%) do masculino. Na faixa de 61 a 70 anos, foram registrados, respectivamente, $112(77,8 \%)$ e $32(22,2 \%)$ prontuários do sexo feminino e masculino, totalizando 144 (17,02\%). Por fim, foram encontrados $44(73,3 \%)$ do sexo feminino e $16(26,7 \%)$ do masculino, totalizando $60(7,1 \%)$ prontuários na faixa etária de 71 a 80 anos. Na categoria "não consta", foram encontrados $8(0,9 \%)$ prontuários, sendo $7(87,5 \%)$ do sexo masculino e apenas $1(12,5 \%)$ do masculino.

A concomitância de sintomas psicológicos à queixa de vertigem foi verificada em 477 (56,38\%), de um total de 846 prontuários. Desse total, $362(75,9 \%)$ prontuários foram de pacientes do sexo feminino, na faixa etária de 51 a 60 anos e $115(24,1 \%)$ do sexo masculino, na mesma faixa etária. Dentre os sintomas psicológicos relatados (angústia, ansiedade, medo, depressão e distúrbios de memória), a angústia foi o de maior prevalência, relatada em 226 (47,38\%) do total de 477 prontuários, sendo 169 (35,43\%) de pacientes do sexo feminino e 57 (11,95\%) do masculino. A ansiedade foi o segundo sintoma mais freqüentemente associado à queixa de vertigem, registrada em 94 (19,71\%) prontuários, sendo $73(15,3 \%)$ de pacientes do sexo feminino e 21 $(4,40 \%)$ do masculino. O medo foi relatado por 46 (9,64\%) pacientes do sexo feminino e por $18(3,77 \%)$ do masculino, perfazendo $64(13,42 \%)$ do total de 477 prontuários. A depressão associada à queixa de vertigem foi registrada em 60 $(12,58 \%)$ prontuários, sendo $49(10,27 \%)$ de pacientes do sexo feminino e $11(2,3 \%)$ do masculino. Por último, os distúrbios de memória, constatados em 33 (6,92\%) prontuários, sendo $25(5,24 \%)$ de pacientes do sexo feminino e 8 (1,68\%) do masculino.

\section{DISCUSSÃO}

Os resultados obtidos apontam para a associação entre a queixa de vertigem e sintomas psicológicos em 56,38\% dos casos, enquanto a literatura aponta para esta associação em 46,5\%, com a prevalência da depressão (14,1\%), ansiedade e medo $(10,3 \%)^{1}$. A presença da ansiedade, da depressão e do medo como sintomas psicológicos concomitantes à vertigem foi devidamente salientada pela literatu$\mathrm{ra}^{1,5-12}$. A angústia, porém, surge como novo fator a ser considerado no diagnóstico de pacientes otoneurológicos com queixa de vertigem.

A otoneuropsicologia considera a vertigem como uma das principais manifestações da angústia e como seu equivalente somático ${ }^{14}$. A angústia, bem como a ansiedade, se originam do ódio, mas do ponto de vista evolutivo, a angústia é anterior à ansiedade ${ }^{13}$. É nesta perspectiva que a angústia, bem como a ansiedade, a depressão e o medo deve ser considerada como manifestação concomitante à verti- 
gem, no diagnóstico, prognóstico e terapêutica dos distúrbios do equilibrio. Deste modo, o sintoma psíquico é a representação atual de conflitos do passado, vivenciados de forma traumática e podendo ser reativados, explicando o aparecimento da vertigem ${ }^{13}$. Se um transtorno psíquico pode ser representado por uma crise vertiginosa ${ }^{4}$, coloca-se a questão: a angústia não poderia ser o representante psíquico da vertigem?

\section{CONCLUSÃO}

Os sintomas psicológicos concomitantes à queixa de vertigem em prontuários de pacientes otoneurológicos foram, em ordem decrescente, a angústia, a ansiedade, o medo, a depressão e os distúrbios de memória. Dentre estes, a angústia foi o sintoma psicológico de maior prevalência.

\section{REFERÊNCIAS BIBLIOGRÁFICAS}

1. Caovilla HH, Ganança MM, Munhoz MSL, Silva MLG \& Frazza MM. O equilíbrio corporal e seus distúrbios: lidando com o paciente vertiginoso. Revista Brasileira de Atualização em Otorrinolaringologia 1997; 4(2):47-51.

2. Ganança MM, Caovilla HH, Munhoz MSL, Silva MLG, Ganança FF, Ganança CF. A vertigem explicada: diretrizes diagnósticas. Revista Brasileira de Atualização em Otorrinolaringologia. Caderno de Vertigem 1999; 2 (56):2-20.

3. Ganança MM, Caovilla HH, Munhoz MSL, Silva MLG, Ganança FF, Ganança CF. Labirintopatias: como diagnosticar e tratar. Revista Brasileira de Atualização em Otorrinolaringologia 1999; 6(1):312 .
4. Haralanov S, Claussen CF, Schneider D. Evaluation of subjective vestibular symptoms: a problem on the borderline between neurootology and psychiatry. Neurootology Newsletter 2000; 5(1):7-11.

5. Sullivan M, Clark MR, Katon WJ, Russo J, Dobie RA, Voorhees R. Psychiatric ad otologic diagnoses in patients complaining of dizziness. Arch International Medicine 1994; 154(5):590-4.

6. Monzani D, Casolari L, Guidetti G, Rigatelli M. Psychological distress and disability in patients with vertigo. Journal of Psychosomatic Research 2001; 50(6):319-23.

7. Jacob RG, Furman JM. Psychiatric consequences of vestibular dysfunction. Current Neurology 2001; 14(1):41-6.

8. Grunfeld EA, Gresty MA, Bronstein AM, Jahanshahi M. Screening for depression among neuro-otology patients with and without identifiable vestibular lesions. International Journal of Audiology 2003; 42(3):161-5.

9. Yardley L, Redfern MS. Psychological factors influencing recovery from balance disorders - Journal of Anxiety Disorders 2001; 15(12):107-19.

10. Yardley L. Contribution of symptoms and beliefs to handicap in people with vertigo: a longitudinal study. Journal of Clinical Psychology 1994; 33(Pt 1):101-13.

11. Hallam RS, Hinchcliffe R. Emotional stability, its relationship to confidence in maintaining balance. Journal of Psychosomatic Research 1991; 34(4-5):421-30.

12. Jacob RG, Whitney SL, Detweller-Shostak G, Furman JM. Vestibular rehabilitation for patients with agoraphobia and vestibular dysfunction: a pilot study. Journal of Anxiety Disorders 2001; 15(1-2):131-46.

13. Kuhn AMB. Das implicações psicológicas nos distúrbios da audição e do equilíbrio corporal [Livre Docência] - Universidade Federal de São Paulo - Escola Paulista de Medicina, 2002.

14. Kuhn AMB, Bocchi EA, Pasquali MAS, Lunedo S. A vertigem na Otoneuropsicologia. Acta AWHO 2001; 20(4):221-3.

15. Manual Diagnóstico e Estatístico de Transtornos Mentais. 4⿳a ed Porto Alegre: Artes Médicas Sul; 2000. 